\title{
Laboratory program on Fourier spectroscopy
}

Marina Bagenova, Lidia Luizova

Marina E. Bagenova, Lidia A. Luizova, "Laboratory program on Fourier spectroscopy," Proc. SPIE 1603, Education in Optics, (1 March 1992); doi: $10.1117 / 12.57899$

SPIE. Event: Education in Optics, 1991, Leningrad, Russian Federation 


\title{
Laboratory program on Fourler spectroscopy
}

\author{
M.E.Bagenova, L. A. Luizova \\ Petrozavodsk State University, Department of Physics \\ Petrozavodsk 18564Ø, USSR
}

\begin{abstract}
A description of laboratory work an Fourier spectroscopy in visible spectral region is given.Students aquire an experience on proceeding with interfercmeter, pressure scanning device, computer data aquisition system and corresponding software. They calculate and determinate spectral qualities of instrument in practice.

\section{INTRODUCTION}

Multiplicity of publications acknowledge rapid development of the Fourier transform spectroscopy for the last 10 years. Many universities introduced the Fourier transform spectroscopy into the curriculum of modern physics. The laboratory curriculum on the Fourier transform spectroscopy is distinguished by a high level of automatization to meet the demand of the Fourier transform spectroscopy method using the spectrum numerical reconstruction from interferogram. As a rule programs in the Fourier transform spectrometer operation system composition are mastered by a user easily, therefore they are convenient in student teaching.

We are giving an abridged description of the laboratory program at the breadboard of the individual construction Fourier transform spectrometer for visible spectrum. Fulfiling the laboratory program students acquaint themselves with the fundamental principles of the Fourier transform spectroscopy, master the technique of the spectrum production at the Fourier transform spectrameter breedboand, calculate and determine spectroscopical characteristics of the device in practice.
\end{abstract}

\section{BRIEF THEORY}

The methods of a Michelson interfercmeter application for the unmonochromatic radiation spectral composition research is called as a Fourier spectroscopy and differ from a classical method by an absence of radiation spectral decomposition 1,? One can briefly describe the Fourier spectrometer model this way: the interferameter is lit up by the radiation under investigation, and the receiving outlet curve is subjected to Fourier analysis and as a consequence is transformed into the distribution of intensity along a frequency. In certain cases such complicated methods is proved more effective than a direct spectrum anslysis, since the Fourier spectrometer is one of the best optical devices for such purpose. The Fourier spectrameter advantages is a high resolving power, a relative aperture increase, and a most considerable increase of information capacity ${ }^{1}$.

The Fourier spectrometer optical unit has been constructed for the flow of radiation under investigation to be forked by a beam splitter into two waves, which after the passage of different optical distance interfere and hit a photodetector. 
A photodetector indicate the signal depending on the change of the optical path difference, which is called an interferogram.

Interferogram can be represented as

$$
F(\Delta)=\int_{\sigma_{\operatorname{mix}}}^{\sigma_{\max }} \mathrm{q}(\sigma) p(\sigma) \cos (2 \pi \sigma \Delta) d \sigma
$$

where $\triangle$ is the optical path difference, $\sigma$ is the navenumber, $q(\sigma)$ is the source radiation spectral distribution, $p(\sigma)$ is the photodetector spectral response, $\sigma_{\max }$ and $\sigma_{\mathrm{min}}$ are the maximum and minimum navenumber of the spectrum of radiation under investigation, which can be recorded by a photodetector, respectively.

Conducting the inverse Fourier transform operation of the interferogram and knowing the photodetector response curve one can calculate a spectrum

$$
\mathrm{q}(\sigma)=\frac{-1}{\mathrm{p}(\sigma)}-\Phi^{-1}\{F(\Delta)\}
$$

where $\Phi_{-1}$ is the convention of the inverse Fourier transform operation.

\section{TASK POFaLdaTION}

The laboratory program task is the production of the filter transmission coefficient spectral distribution curve represented in the form of a graph printed out by a printer.

Solving of this task consists of a few stages.

1. Fourier spectrometer alignment:

a) production of the fringe pattern from a laser;

b) production of the contrasting fringe pattern from an incandescent lamp;

c) plotting of the lamp radiation symmetric interferogram by a recoder;

d) plotting of laser radiation interferogram by a recoder;

2. Interferograms recording and data processing:

a) interfercgram recording and writing it down on the diskette are performed under the control of "Interferogram recording" program;

b) interferogram processing and spectrum reconstruction are performed under the control of the "Fourier spectrometer" program;

c) writing on one's own a program for the filter transmission spectral coefficient determination;

d) placing the transmission curve graph into a printer by means of "Spectrum construction" program.

\section{APPARATOS}

A block diagram of a experimental apparatus is show in Fig.1. The optical unit of a breadboard has been assembled on the base of the holographic apparatus by a Michelson interferometer circuit. Going through a semitransparent beam splitter the beam of the radiation under investigation breaks into two beams, which are reflec- 


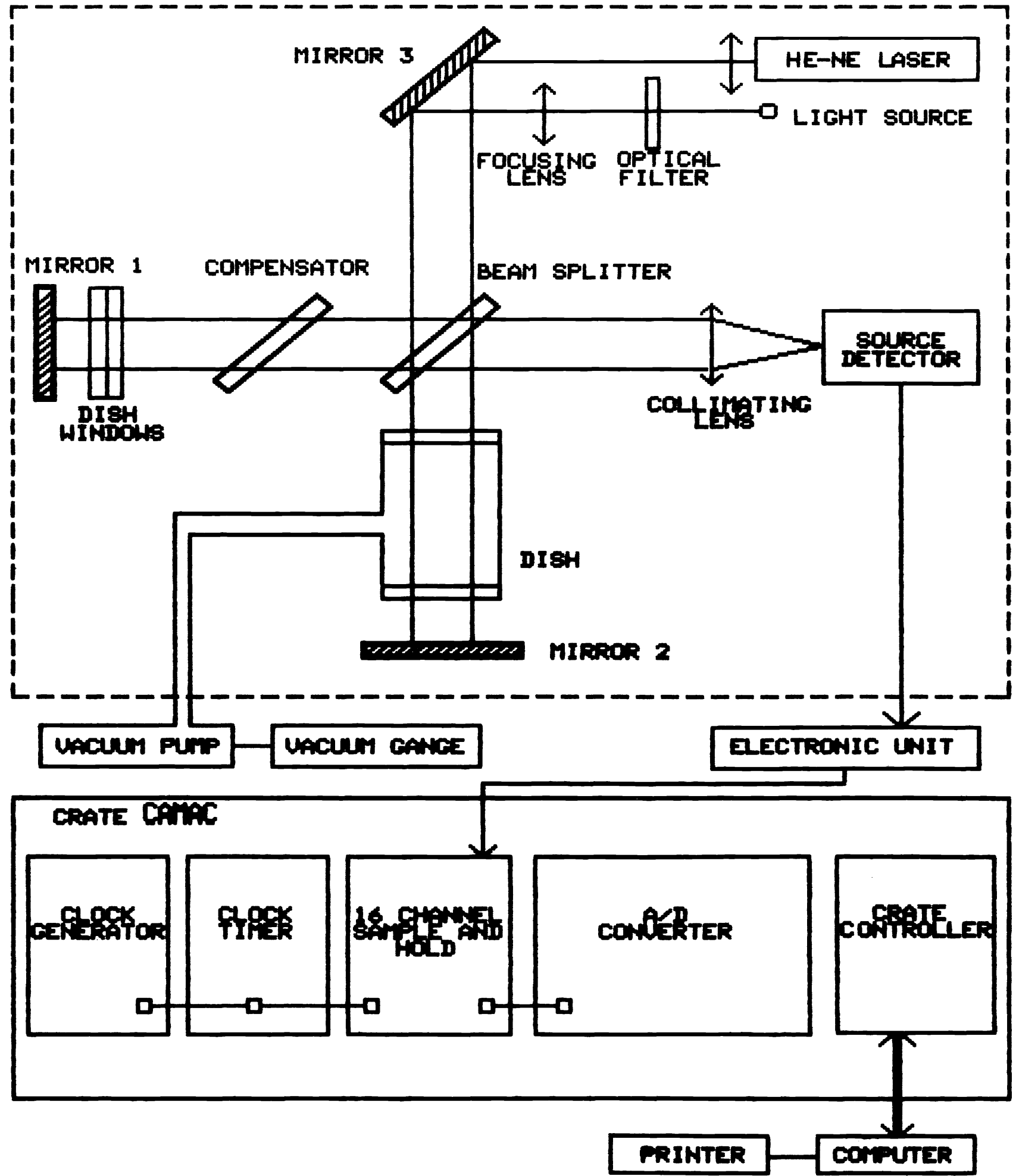

Fig. 1. Block diagram of the Fourier transform spectrometer breadboard. 
ted from mirrors and interfere; a fringe pattern comes out in the form of rings. Scanning is done by an optical method, with a change procedure of an index of refraction of the medium the beam passes through. For that purpose the glass dish connected with a vacuum purm is placed in the beam path. Since refractive index of air depends on pressure, the path difference between interfering beams changes during evacuation. The dish windows are placed for compensation of the path difference additional change appearing when the beam passes through the dish windows.

For recording of the fringe pattern rings intensity the optical signal is bound for a photodetector connected over the electronic unit and analog memory multichannel device with the $\mathrm{AD}$ converter of the crate CAMAC. $\mathrm{AD}$ converter converts the electric signal in the numeric code and array we call the data filled in this way as the source interferogram. At the present apparatus the scarning along the beam path difference is realized by the method of the unbroken slow bleeding-in the air into the dish through a cappllary. A recording system allows to make the indications across planed step, but it is impossible to tie them with a path difference definite value. Therefore for the path difference determination at the received

interferagram of a source the He-Ne laser radiation interferogram is recorded simultaneously ( the reference interferogram).

\section{METHOD IDEA}

The method idea of the filter transmission spectral coefficient $T(\lambda)$ determination lies in the consecutive recording of two interferograms - at first the radiation of an incandescent lamp without a filter (see, for example, Fig.2a), then into the radiation bundle introduces the filter under investigation (see, for example, Fig.2b).

As a result of the computer processing of these interferograms we receive for the first experiment - the lamp radiation spectral distribution $q(\lambda)$ (see, for example, Fig. 3a), for the second experiment - the spectral distribution $q(\lambda) \tau(\lambda)$ of the lamp radiation through a filter (see, for example, Fig. 3b).

Dividing the second result into the first result we receive the demanded distribution (see, for example, Fig.3c).

\section{CONTROL QUESTIONS}

Control questions are offered to students after fulfilment of experimental part of the laboratory program. Considering the answers students learn to employ their theoretical knowledge for practical problems of the experiment.

1. What is the purpose of laser radiation interferogram recording ?

2. How is the optical path difference defined by laser radiation interferogram ?

3 . What is the aim of placing windows without dish and compensator in the second arm of the interferometer ? Why can't the increase of the beam optical path in the dish windows of the first arm be compensated with simple movement of the second arm mirror ?

4. What does the contrast depend on ? 
5. How does spectrum width influence the interferogram appearance ?

6. How is a step along interferagram for spectrum reconstruction connected with radiation spectral range?

7. What parameters of the device and recording system influence the resolution and spectrum maximum width, which can be recorded ?

\section{REFERENCES}

1. R.J.Bell. Introductory Fourier transform spectroscopy, Moscow, Mir, 1975.

2. Tolmachev U.A. New spectral devices, Leningrad university, 1976.

3. Moskalev V.A. Theoretical bases of optophysical investigations, Leningrad, Mashinostroenie, 1987.
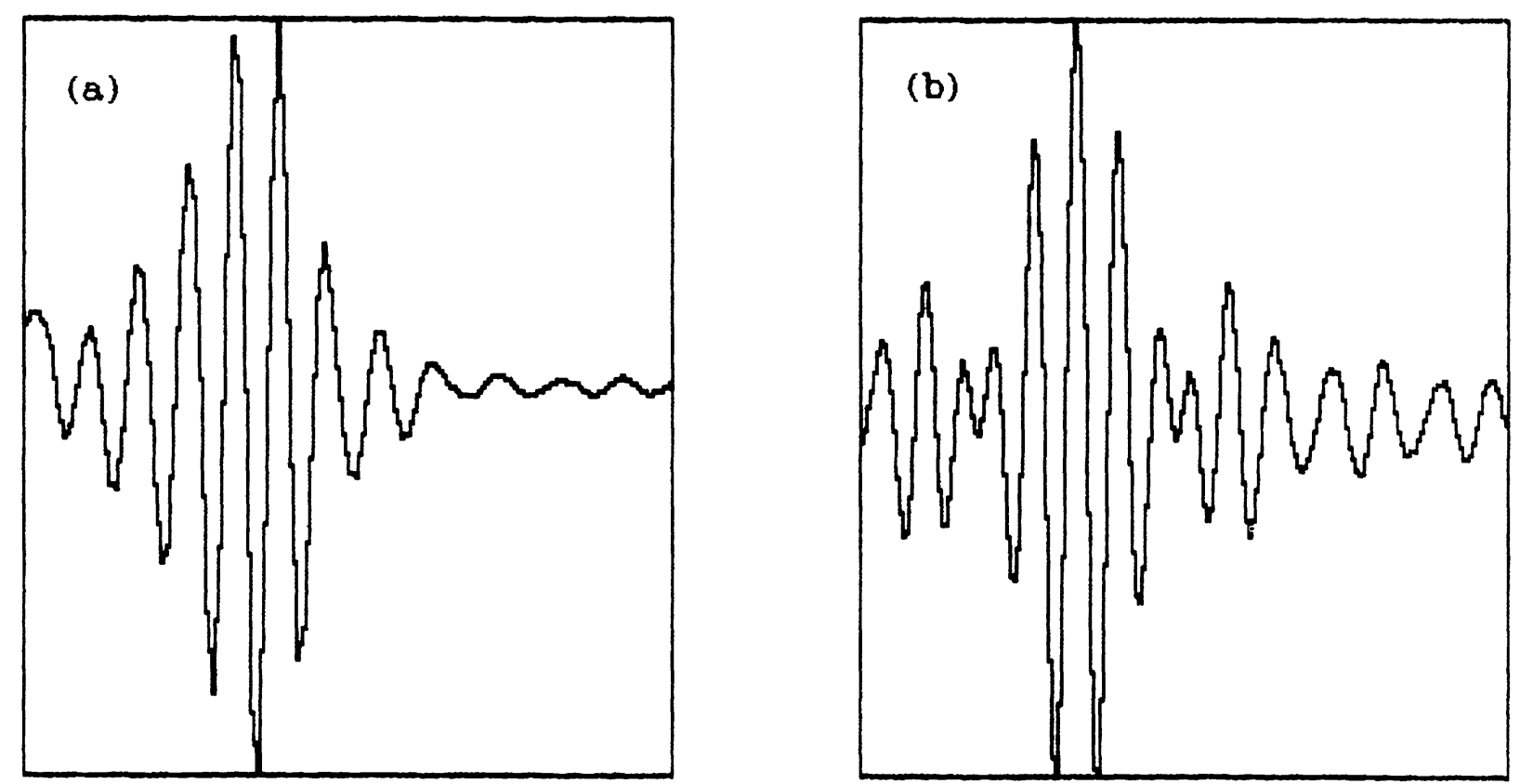

Fig.2. Experimental results of interferograms measurement:

(a) the interferogram of a incandescent lamp;

(b) the interferogram of the same lamp as in a but through the glass 

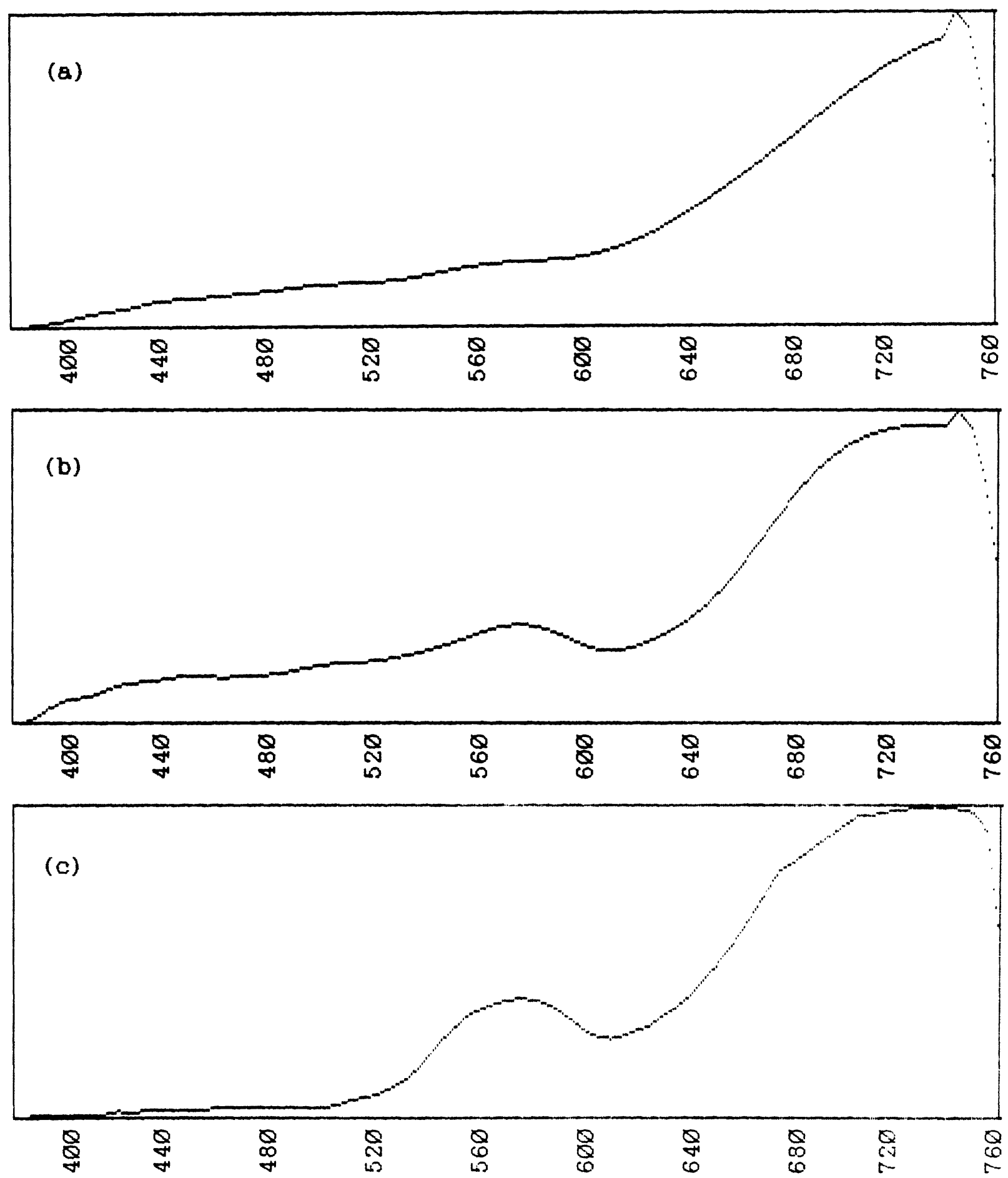

Fig.3. Experimental results of transmittance spectra measurement :

(a) the background spectrum of a incandescent lamp;

(b) the spectrum of the same lamp as in a but through the glass filter;

(c) the reconstructed transmittance spectrum of glass filter from a and b. 